\title{
BMJ Open Evaluation of long-term clinical and health service outcomes following coronary artery revascularisation in Western Australia (WACARP): a population-based cohort study protocol
}

\author{
C Gardner, ${ }^{1} \mathrm{~J}$ M Rankin, ${ }^{2}$ E Geelhoed, ${ }^{1}$ M Nguyen, ${ }^{3}$ M Newman, ${ }^{4}$ D Cutlip, ${ }^{5}$ \\ M W Knuiman, ${ }^{1}$ T G Briffa, ${ }^{1}$ M S T Hobbs, ${ }^{1}$ F M Sanfilippo ${ }^{1}$
}

To cite: Gardner C,

Rankin JM, Geelhoed E, et al. Evaluation of long-term clinical and health service outcomes following coronary artery revascularisation in Western Australia (WACARP): a population-based cohort study protocol. BMJ Open 2014:4:e006337.

doi:10.1136/bmjopen-2014006337

- Prepublication history for this paper is available online. To view these files please visit the journal online (http://dx.doi.org/10.1136/ bmjopen-2014-006337)

Received 8 August 2014 Accepted 19 August 2014

\section{CrossMark}

For numbered affiliations see end of article.

Correspondence to Assoc Prof Frank Sanfilippo; frank.sanfilippo@uwa.edu.au

\section{ABSTRACT}

Introduction: Coronary artery bypass grafting (CABG) and percutaneous coronary interventions $(\mathrm{PCl})$ are procedures commonly performed on patients with significant obstructive coronary artery disease to relieve symptoms of ischaemia, improve survival or both. Although the efficacy of both procedures at the individual level has been established, the impact of advances in coronary artery revascularisation procedures (CARP) on long-term outcomes and cost-effectiveness at the population level are yet to be assessed. Our aim is to evaluate a minimum of 6-year outcomes and costs for the total population of patients who had CARP in Western Australia (WA) in 2000-2005.

Methods and analysis: This retrospective population cohort study will link clinical and administrative health data for a previously defined cohort including all patients in WA who had a CARP in the period 2000-2005. The cohort consists of 19014 patients who had 21175 procedures (15 $429 \mathrm{PCl}$ and $5746 \mathrm{CABG}$ ). We are now collecting a minimum of 6 years follow-up of morbidity and mortality data for the cohort using the WA Data Linkage System, clinical registries and hospital records, with 12 years follow-up for cases in the year 2000. Comparison of long-term outcomes for different CARP will be reported ( $\mathrm{PCl}$ vs $\mathrm{CABG}$; bare metal stents vs drugeluting stents vs $(A B G)$. Cost-effectiveness analysis of CARP from the perspective of the healthcare sector will be performed using individual level cost data and average costs from Australian Refined Diagnosis Related Groups.

Ethics and dissemination: This study has received ethics approval from the University of Western Australia, the Western Australian Department of Health and all participating hospitals. Being a large population cohort study, approval included a waiver of informed consent. All findings will be presented at local, national and international healthcare/academic conferences and published in peer-reviewed journals.

\section{INTRODUCTION}

Coronary artery bypass grafting (CABG) and percutaneous coronary intervention (PCI) are established methods of mechanical

\section{Strengths and limitations of this study}

- A comparison of trends for a minimum of 6-year clinical outcomes following bypass surgery, bare metal stents and drug-eluting stents will be the first such study in the total coronary artery revascularisation population.

- Linkage of routinely collected state-wide health administrative data with clinical registries allows the ascertainment of accurate and complete data on long-term outcomes for the cohort.

- This study avoids many limitations of clinical trials including restricted patient samples, short follow-up periods and relatively small numbers, and will provide valuable information about patients typically excluded from clinical trials.

- Given that this is an observational cohort study, there is potential for bias due to the nonrandomised assignment to a treatment, but this will be addressed through propensity score methods.

revascularisation for coronary artery disease, yet their comparative effectiveness in terms of long-term clinical outcomes and costeffectiveness remains unclear. ${ }^{1-3}$ PCI is a less invasive method than CABG and initially involved the use of balloon angioplasty in the 1980s followed in the 1990s by the introduction of coronary artery stents. Bare metal stents (BMS) were used initially, and in Western Australia (WA), these were largely replaced by drug-eluting stents (DES) from mid-2002. DES devices have a slow-release drug with antimitotic drug properties embedded in the stent matrix to reduce the risk of stent restenosis compared with BMS. Collectively, CABG and PCI are referred to as coronary artery revascularisation procedures (CARP).

A major disadvantage of PCI compared with CABG is its higher rates of repeat 
revascularisation due to restenosis in treated vessels, and this has contributed to the rise in CARP rates. Repeat procedures within 12 months were required in about $30 \%$ of balloon angioplasty cases, compared with $15 \%$ for BMS and $5 \%$ for DES procedures. ${ }^{45}$ Pivotal clinical trials have shown superiority at 12 months for DES over BMS in reducing in-stent restenosis and hence target vessel revascularisation (TVR: treatment of the same vessel that was treated previously). ${ }^{6}{ }^{7}$ Concerns about the long-term safety of DES were raised with several reports at the European Society of Cardiology meeting in 2006 suggesting increased late mortality possibly due to late stent thrombosis. ${ }^{67}$ A later report from a large registry study in Sweden (SCAAR study group) noted similar findings. ${ }^{8}$ Subsequent studies applying uniform definitions for late stent thrombosis from the Academic Research Consortium $^{9}$ suggested a slight increase in risk for stent thrombosis beyond 1 year (very late stent thrombosis) for DES, but no increase in overall stent thrombosis or mortality rates. ${ }^{8}{ }^{10-12}$ Parallel results from multiple large registries, including a more complete follow-up from the Swedish registry, have also indicated no increase in mortality for DES compared with BMS. ${ }^{13-15}$ However, the issue of very late stent thrombosis remains a concern. ${ }^{16}$ Despite the worldwide use of DES in millions of patients to reduce the risk of TVR, little is known of the rates and risk factors for late stent thrombosis and how these vary over time. Complicating the real-world impact of very late stent thrombosis associated with DES is the widespread use of DES in off-label situations, variation and duration of antiplatelet therapy and inadequate follow-up periods in clinical trials to quantitate the risk of early stent thrombosis ( $\leq 30$ days), late stent thrombosis (31-365 days) and very late stent thrombosis ( $>1$ year). It also appears that the mechanisms leading to very late stent thrombosis are distinct from those responsible for early or late stent thrombosis. ${ }^{17}$ First generation DES releasing sirolimus or paclitaxel have a steady annual risk of stent thrombosis of $0.3-0.6 \%$ per year for at least 5 years. ${ }^{16} 1819$

CARP constitute a significant component of the health costs of coronary heart disease (CHD), which consumes approximately $3 \%$ of total healthcare expenditure in Australia. ${ }^{20}$ While the efficacy of CARP has been demonstrated through clinical trials and meta-analyses, the impact of advances in CARP on the adverse long-term outcomes of cardiovascular disease (CVD), health service utilisation, health costs and cost-effectiveness has not been assessed in the population. Nor have advances in revascularisation technology, particularly DES, been evaluated in terms of clinical outcomes and cost-effectiveness within the total population undergoing coronary revascularisation.

Assessment of the comparative effectiveness and outcomes in populations undergoing CARP from case series, registries and randomised controlled trials (RCTs) is difficult and the generalisability of results to the general population can be problematic. Most studies examine either PCI or CABG, although in some cases direct comparisons between different revascularisation procedures are carried out. ${ }^{1}{ }^{21} 22$ Results from RCTs frequently suffer from poor generalisability because of the highly selective nature of patients who participate in trials. Many RCTs exclude patients with cardiogenic shock, left main coronary artery disease, concomitant valvular disease and severe complex lesions, unsuitable for stenting, but who would have benefited from surgery. ${ }^{23}{ }^{24}$ To a large extent, these studies exclude patients with more severe CHD who have shown a survival benefit from surgery, effectively biasing these trials against the prognostic benefit of CABG. ${ }^{25}$ Furthermore, despite evidence of the continued risk of stent thrombosis and other long-term adverse events, many clinical trials have short follow-up periods of insufficient length to capture important clinical events. In addition, pivotal RCTs that have informed clinical decision-making have relatively small sample sizes and were underpowered to detect rare events such as stent thrombosis.

The WA Data Linkage System ${ }^{26}$ provides a unique opportunity to examine the long-term outcomes of CARP in a large population by linking routinely collected statewide health administrative data with clinical registries for all CARP performed in WA. We will assess the long-term comparative effectiveness of CARPs in the total population of patients undergoing revascularisation procedures. We will also examine the costs and cost-effectiveness of CARP in the context of a wide range of covariates.

\section{OBJECTIVES}

An established population cohort of WA residents who had a CARP in the period 2000-2005 will be used to examine the long-term outcomes and costs using data from the WA Data Linkage System, clinical registries, patient level costs and hospital notes. The objectives of the study are:

1. To evaluate the long-term (6 years for the entire cohort and up to 12 years follow-up for varying fractions of the cohort) clinical outcomes following the first CARP in WA adjusting for diagnosis, type of procedure and demographic characteristics including age, gender, socioeconomic status, location and Aboriginal status.

2. To describe the epidemiological and clinical determinants of long-term trends in health service utilisation and costs following first admissions for CHD, including the impact of changing revascularisation technology and medical treatment.

3. To determine the long-term cost-effectiveness of different CARP (BMS vs DES vs CABG, and PCI vs CABG).

\section{METHODS}

\section{Study cohorts}

In WA (population 1.95 million in 2003), CARP are performed exclusively in three adult teaching hospitals and four private hospitals, all located in the Perth metropolitan area. We have previously collected a detailed data set on all patients who had a CARP at any of these seven 
hospitals during 2000-2005 for a study evaluating the impact of the introduction of DES on short-term outcomes following CARP. ${ }^{27}$ The current study runs from 2011 to 2015 and will use this cohort (WACARP cohort) to obtain data on outcomes and costs from long-term follow-up to be applied in analyses of clinical outcomes and cost-effectiveness. The WACARP cohort consists of 19014 patients who had 21175 procedures (5746 CABG and 15429 PCI) during 2000-2005 (see table 1).

The Hospital Morbidity Data Collection (HMDC) is one of the core data sets of the WA Data Linkage System ${ }^{26}$ and consists of all admissions to every hospital in WA (public and private) since 1980. Diagnoses and procedures are coded on discharge using the International Classification of Diseases (ICD) manuals, and represent discharge diagnoses rather than presenting conditions. Coding at discharge is a distinct advantage in that the use of admission diagnosis to define an admission as CHD-associated is problematic since patients often have diagnoses that change during admission as a result of additional clinical consideration and investigation. We have obtained from the HMDC an extract of all cardiovascular admissions since 1980. The PCI and CABG admissions in the WACARP cohort were identified from this extract using procedure codes from the ICD 10th revision Australian Modification (ICD-10-AM). Codes were: (1) 35304-00, 35305-00 (balloon angioplasty); (2) blocks 669 and 671 (angioplasty with stents) and (3) blocks 672-679 (CABG).

The WACARP cohort has been linked to all corresponding records in the HMDC cardiovascular extract and the linkage will be used to identify the first (index) admission for PCI or CABG in the cohort during 20002005 by applying a fixed lookback period of 10 years. Any person in the WACARP cohort who has an admission for PCI or CABG in the previous 10 years will be excluded to produce the Index WACARP Cohort. Index cases will be classified as either PCI (BMS or DES) or CABG. If a patient had both a PCI and CABG in their index admission, we will classify them according to which procedure came first. All patients in the cohort will be followed identically regardless of CARP status. Patients who were non-WA residents (identified by residential postcode from the HMDC) will be excluded from the index cohort.

In addition, the HMDC extract of cardiovascular admissions will allow us to identify the total cohort of people aged from 25 years ever admitted to hospital with a discharge diagnosis of CHD (ICD-9-CM codes 410-414, ICD-10-AM codes I20-I25) in any diagnosis field in the period 1980-2010 (Total CHD cohort).

\section{Data sources and ongoing collection of outcome and covariate variables}

This study is adding to data collected previously for the WACARP cohort in order to assess long-term outcomes following a CARP. Data will come from both clinical sources (eg, registries) and from the WA Data Linkage System (HMDC extract of cardiovascular admissions and death record). Clinical data for PCI procedures are being obtained from the seven CARP hospitals. These include electronic data from registries maintained by cardiology departments at the three teaching hospitals (collection completed), as well as the ongoing data collection from medical notes of the private hospitals. Electronic clinical data for the CABG cases have been obtained from registries maintained by departments of cardiothoracic surgery and anaesthesia (perfusion) at the teaching hospitals. Information about CABG performed privately (40\%) was unable to be obtained. Missing or inconsistent data from electronic sources were queried with data custodians from the corresponding hospital, and amended where possible. All of these data sets (electronic and notes) will be linked to their corresponding HMDC and death records using the patient's unique medical record number and procedure date as the linkage fields.

Clinical details of PCI procedures from private hospitals are collected from billing sheets, medical notes (hospital and/or cardiologist notes), the radiographer's journal, nursing intervention records, as well as the cardiologist's procedure notes. The aggregated minimum data set of PCI and CABG cases includes: residential postcode, age, gender, admission and discharge dates, type of hospital, type of admission, discharge diagnosis and procedure codes (for current diagnosis and medical history including

Table 1 Number (and percentage of total CARP) of revascularisation procedures in the total WACARP cohort of 2000-2005

\begin{tabular}{|c|c|c|c|c|c|c|c|}
\hline \multirow[b]{2}{*}{ Type of procedure } & \multicolumn{6}{|c|}{ Year of procedure } & \multirow[b]{2}{*}{ Total } \\
\hline & 2000 & 2001 & 2002 & 2003 & 2004 & 2005 & \\
\hline BMS only & 1798 (56.1) & $1942(60.9)$ & 1563 (46.1) & 412 (11.4) & $116(3.0)$ & 114 (2.9) & 5945 \\
\hline DES & 0 & 0 & 627 (18.5) & 2145 (59.5) & 2795 (72.3) & 2865 (73.2) & 8432 \\
\hline Total stents & 1798 (56.1) & $1942(60.9)$ & 2190 (64.6) & 2557 & 2911 & 2979 (76.1) & 14377 \\
\hline POBA/rota & 244 (7. & 169 (5.3) & $126(3.7)$ & $60(1.7)$ & $34(0.9)$ & $54(1.4)$ & 687 \\
\hline Missing PCI type & $54(1.7)$ & $45(1.4)$ & 63 (1.9) & $55(1.5)$ & $58(1.5)$ & $90(2.3)$ & 365 \\
\hline Total PCI & 2096 (65.4) & 2156 (67.6) & 2379 (70.1) & $2672(74.1)$ & $3003(77.7)$ & $3123(79.8)$ & 15429 \\
\hline CABG & 1109 (34.6) & 1034 (32.4) & 1013 (29.9) & 934 (25.9) & 864 (22.3) & 792 (20.2) & 5746 \\
\hline Total CARP & 3205 & 3190 & 3392 & 3606 & 3867 & 3915 & 21175 \\
\hline
\end{tabular}

BMS, bare metal stent; CABG, coronary artery bypass grafting; CARP, coronary artery revascularisation procedures; DES, drug-eluting stent; $\mathrm{PCI}$, percutaneous coronary intervention (stents+POBA/rota); POBA, plain old balloon angioplasty; rota, rotablator (rotational atherectomy). 
comorbidities), procedure date and time, vessels treated, number of vessels grafted, type of grafts used (venous, arterial), number and details of stents inserted (name, brand, diameter, length), and date and cause of death.

These data will be enhanced by linking the following from electronic sources:

1. Results of diagnostic tests from public and private pathology laboratories including type (inpatient/outpatient), troponin $\mathrm{I}$, troponin $\mathrm{T}$, creatine kinase, creatine kinase $\mathrm{MB}$, serum creatinine, fasting glucose, haemoglobin, total cholesterol, high-density lipoprotein, low-density lipoprotein (calculated), triglycerides, estimated glomerular filtration rate, total white cell count, glycosylated haemoglobin;

2. Blood products (packed red blood cells, whole blood, fresh frozen plasma, platelets and cryoprecipitate including quantity and date issued) used to treat bleeding complications following CARP (obtained from the transfusion medicine databases at hospitals);

3. Discharge and outpatient medications recorded in electronic hospital pharmacy databases (drug, dose, directions, date dispensed, quantity);

4. Presence of stent thrombosis from long-term follow-up.

\section{Follow-up and outcomes}

Outcomes will be ascertained from a combination of HMDC and death records, hospital notes and electronic sources stated above. Follow-up events over 6 years for index CARP cases will include: (1) death (CVD and all-cause); (2) hospital admission for myocardial infarction (MI); (3) hospital admission for TVR; (4) hospital admission for stroke; (5) composites: death or MI, death or TVR, death or MI or TVR, death or MI or TVR or stroke and (6) stent thrombosis according to the Academic Research Consortium definition. ${ }^{9}$ For index PCI cases, a TVR end point is defined as a subsequent PCI procedure in the same vessel as in the index PCI (as identified from clinical data from notes or electronic sources), or as any subsequent CABG procedure (identified from HMDC), whichever was first. For index CABG cases, a TVR end point is any subsequent PCI or CABG procedure (identified from HMDC). Two analyses will be undertaken. For the first, follow-up will cease on the date of an outcome/end point within 6 years of the index date or at the end of the 6-year period. Patients will be censored if the outcome does not occur during follow-up. A separate analysis will be performed using as much follow-up time as is available to 12 years.

\section{Stent thrombosis}

Our large number of PCI cases $(n=15429)$ will allow evaluation of the frequency and predictors of early ( $\leq 30$ days), late (31 days to 1 year) and very late $(>1 \text { year })^{9}$ stent thrombosis and its impact on mortality and MI outcomes for BMS compared with DES. Follow-up for very late stent thrombosis will be for at least 6 years and up to 12 years. Cases to review will be identified from the linked HMDC and death data sets. Data to determine the presence of stent thrombosis will be collected by cardiology fellows/trainees from angiogram films stored electronically or in medical notes at each of the seven hospitals mentioned previously and entered into an online database. Stent thrombosis will be classified as definite, probable, possible and not present according to the Academic Research Consortium definition. ${ }^{9}$

\section{Comorbidities}

The HMDC will be used to identify previous admissions within 5 years of the index CARP where the discharge diagnosis was recorded as MI, stroke, diabetes, renal failure, congestive heart failure, peripheral vascular disease or chronic obstructive pulmonary disease in any diagnosis field. Similarly, a Charlson comorbidity score ${ }^{28}$ will be calculated as a composite measure of comorbidity for each index case by applying a fixed 5-year lookback period using the HMDC. We will use the Dartmouth-Manitoba ICD code assignments ${ }^{29}$ in calculating the Charlson score based on the original 17 Charlson comorbidities.

\section{Socioeconomic status and residential location}

The HMDC includes variables for measuring socioeconomic status and residential remoteness by supplying values for Socio-Economic Indexes for Areas (SEIFA) ${ }^{30}$ and the Accessibility/Remoteness Index of Australia $(\text { ARIA }+)^{31}$ based on census years beginning from 1996. These are derived from the residential address and the SEIFA will be used to measure quintiles of socioeconomic disadvantage, while ARIA+ will be used as a measure of geographical disadvantage grouped into five categories (major cities, inner regional, outer regional, remote and very remote).

\section{Estimation of resource use and costs}

Only direct health system costs will be considered, to reflect the perspective of the health sector, ${ }^{32}$ including hospital admissions and selected medical treatments shown to be protective against adverse CVD events in clinical trials. Resource costs have been obtained from teaching hospitals for individual patient admissions and include ward costs; pharmaceuticals; procedural costs; and intensive care, theatre and staff salaries. In addition, the HMDC includes the Australian Refined Diagnosis Related Group (AR-DRG) code for each admission. From this, we can obtain the corresponding AR-DRG cost ${ }^{33}$ which is the average cost of admissions with the same AR-DRG.

\section{General data analysis}

Outcomes will be compared between: (1) PCI versus CABG; (2) BMS versus DES, BMS versus CABG, DES versus CABG; (3) pre-DES period (1 January 2000 to 30 June 2002) versus DES period (1 July 2002 to 31 December 2005; table 2) and (4) calendar year of index 
admission 2000-2005 for each treatment group (DES, BMS, CABG, total PCI, total CARP). Comparisons will be repeated by stratifying by: (1) Aboriginal status; (2) single vessel disease versus multivessel disease; (3) quintiles of socioeconomic disadvantage from SEIFA scores and (4) residential location from ARIA+ categories.

Kaplan-Meier survival curves will be used to compare time to outcome events in the Index WACARP Cohort. Log-rank statistics will be used to test for crude differences. Cox proportional hazards regression models will be used to compare groups after adjustment for covariates including: (1) age; (2) sex; (3) Charlson score, and previous comorbidity (as separate binary variables) for any form of CVD, diabetes, chronic renal failure and chronic obstructive pulmonary disease noted in the 5 years prior to the index admission; (4) diagnosis at index admission (MI, not MI); (5) type of index admission (booked, emergency); (6) type of hospital at index admission (public, private); (7) year of index admission; (8) quintiles of socioeconomic disadvantage at index admission; (9) ARIA+ category; (10) Aboriginal status; (11) indication for CARP; (12) results of cardiac biomarkers and other laboratory tests at index admission; (13) details of index CARP (including type of stent used, total number of stents, length of stent); (14) severity of illness (site of revascularisation, number of vessels treated) and (15) use of cardioprotective drugs. Adjusted Cox survival curves for comparison groups will be plotted from outputs of the Cox regression models using the mean for continuous covariates and the value of the reference level for categorical covariates.

In addition to standard confounder adjustment in Cox regression models, we will also adjust for covariates using propensity score methods ${ }^{34}$ to assess the sensitivity of the group comparisons (ie, the estimated HRs) to the form of adjustment for covariates. Standard confounder adjustment may not work well when there is little overlap in distribution of covariates across comparison groups. Adjustment for propensity score performs better in these circumstances. Any variable that may be associated with the propensity of a treatment being selected will be identified via logistic regression methods and the propensity score for each patient obtained from the fitted logistic model. Once the propensity scores have been calculated, outcomes will be analysed by Cox proportional hazards regression with the propensity score and treatment group as independent variables in the model.

\section{Economic analyses}

The effect of total CARP and procedure type (CABG, balloon angioplasty, BMS, DES, coronary angiography, no procedure) within 90 days of the date of first admission will be examined on subsequent 6 -year costs in the index WACARP cohort. Health costs and health service utilisation will be classified as initial (occurring in the first 90 days) and long-term ( $>90$ days to 6 years). The 90-day initial episode will allow for readmissions for booked procedures from the initial clinical event. Previous research has shown that approximately $95 \%$ of CARP performed within 1 year of MI occur within 90 days. ${ }^{35}$ Costs will be further characterised as: (1) cardiac (or complications related to treatment); (2) other CVD and related conditions (diabetes, renal disease, chronic obstructive pulmonary disease) and (3) not CVD or related conditions.

Age-specific trends in first admissions for CHD will be analysed to determine the extent to which overall health utilisation and health cost are attributed to changes in incidence and survival over time. Trends in the prevalence of CARP by age, sex, diagnosis and procedure at 90 days in the population which had a CHD-related admission during 2000-2005 will also be determined to investigate changes in patient selection.

Cost analysis will be undertaken to explore the cost profiles of different service provision, and to assess the impact of parameters such as diagnosis, age, sex, socioeconomic status, Aboriginality, hospital type (public or private), admission type, cardioprotective drug therapy and comorbidity on the total cost of care. This will guide stratification of the cohort into meaningful subgroups for more detailed analyses. Costs will be adjusted to 2013 dollars by inflating the costs from previous years using the corresponding Health Price Index obtained from the Australian Institute of Health and Welfare. Costs and outcomes compared across differing time periods will be adjusted for time preference using a $3 \%$ discount rate. Sensitivity analyses will investigate varying discount rates of $0 \%$ and $5 \%$.

In general, costs are difficult to analyse alongside other parameters such as disease severity and age because of: (1) the distribution of data (data are usually skewed, with a few patients accounting for a disproportionate share of the total cost); (2) problems in dealing with censored data (patients who die do not continue to incur costs) and (3) the nature of cost accrual, which is irregular and may depend on different factors at

\begin{tabular}{|c|c|c|c|c|c|c|c|}
\hline \multirow[b]{2}{*}{ Type of stent } & \multicolumn{6}{|c|}{ Year of procedure } & \multirow[b]{2}{*}{ Total } \\
\hline & 2000 & 2001 & 2002 & 2003 & 2004 & 2005 & \\
\hline BMS only & $1798(100)$ & $1942(100)$ & $1563(71.4)$ & $412(16.1)$ & $116(4.0)$ & $114(3.8)$ & $5945(41.4)$ \\
\hline DES ( \pm BMS $)$ & 0 & 0 & 627 (28.6) & 2145 (83.9) & $2795(96.0)$ & $2865(96.1)$ & $8432(58.6)$ \\
\hline Total stents & 1798 & 1942 & 2190 & 2557 & 2911 & 2979 & 14377 \\
\hline
\end{tabular}


different times. Owing to these issues, the initial analysis of costs will use mean and median values compared by stratifying on important variables including age, sex, disease severity and Charlson score. More sophisticated models including generalised linear models (GLMs) and Cox proportional hazards regression will be used to adjust for covariates and produce results that are directly interpretable (ie, not $\log$ results that have to be back-transformed).

Cox models have been used for economic analyses since cost distributions are frequently complex and difficult to describe parametrically. ${ }^{36}$ Cox proportional hazards models make no parametric assumptions about the specific error distribution. Furthermore, if a patient dies prior to hospital discharge and complete costs are not obtained, the patient will be censored in the model. Although Cox proportional hazards models can deal with both censored and complex cost distributions, irregular cost accrual may lead to bias in survival estimates. Even though the bias may be small, its magnitude cannot be estimated. ${ }^{36}$ GLMs have recently been introduced in cost data analysis and will be the main models, with appropriately specified GLMs modelling the error structure of cost data more closely. ${ }^{37}$ GLMs are particularly attractive for regression of cost data because they provide parametric methods of analysis where a variety of non-normal distributions can be specified and the way covariates act can be altered. GLMs are also advantageous because they make inferences about mean costs directly as is appropriate for health economic decision-making.

Incremental cost-effectiveness ratios (ICERs) will be compared between groups and across measures of severity of disease, and adjusted for age, comorbidity and other covariates at the index admission. Since cumulative costs and effects for CARPs are dependent on the time interval under consideration, costs and effects will be ascertained for a range of time periods: 0-90 days (initial costs), 91 days to 1 year in 90-day survivors, 1-6 years in 1-year survivors and for total 6-year costs. Follow-up of 6 years will capture the most relevant longterm costs and effects of the two procedures. Modelling will be used to extrapolate results to arrive at lifetime cost-effectiveness estimates.

\section{Sensitivity analysis and acceptability}

The robustness of results of the cost-effectiveness analyses to parameter uncertainty will be examined using probabilistic sensitivity analysis incorporating bootstrapping, since it is more powerful than deterministic sensitivity analysis. ${ }^{38}$ Bootstrap methods will be used to estimate the joint distribution of cost and effectiveness differences, and the proportion of replicates with ICERs less than commonly accepted benchmarks applicable to Australia will be presented. Scatterplots showing the joint distribution of incremental costs and incremental effects between comparison groups for each of the main outcomes will be calculated. In line with recommendations by the National Institute for Clinical Excellence, ICERs will also be summarised using acceptability curves. ${ }^{39}$ These represent the probability (ie, proportion of simulations) that a given intervention is more cost-effective than alternative(s) for a range of maximum thresholds of willingness to pay for an additional outcome measure.

\section{Dissemination}

All findings will be presented at local, national and international healthcare/academic conferences and published in peer-reviewed journals. Relevant findings will also be disseminated through the clinical networks of the cardiologists on the research team.

\section{DISCUSSION}

This study addresses two significant issues about the impact of increasing and changing coronary artery revascularisation technology. First, the population analysis of clinical outcomes and cost-effectiveness comparing specific CARP technologies will be of particular interest to clinicians. The second, which examines the costs and possible savings attributable to CARP in the context of a wide range of environmental and medical factors that affect the cost of CARP, will interest medical decisionmakers. Studies such as ours are limited to the few jurisdictions in which population medical record linkage is possible. As revascularisation technologies in Australia during the study period were the same as those available in other countries, the results of our population analysis will be applicable in other jurisdictions throughout the world.

While randomised clinical trials indicate that DES have short-term clinical and health service advantages over BMS, DES are more expensive and their use may therefore be restricted in practice. Moreover, there are still unresolved questions about the possible longer term complications of DES and whether the initial (1-year) benefits of DES are sustained over the longer term. The future of CABG procedures, which have been declining in absolute and relative terms compared with PCI over many years, is also unclear. Finally, there has been no population assessment of the impact of rapid increases in concomitant medical treatment on outcomes of CARP.

This study will compare trends in clinical outcomes following $\mathrm{CABG}$, BMS or DES in a total CARP population over 6 years and up to 12 years. It will use an existing large cohort of $>19000$ patients treated with CARP to show if improved outcomes in one or more of the CARP types will translate into benefits for the total population of recipients and the healthcare sector. The inclusiveness of our cohort (all CARP) and complete ascertainment of data on outcomes and end points during follow-up is a major strength of this study. The ability to link procedural and clinical data with routinely collected administrative data through the WA Data 
Linkage System allows identification of comorbid conditions and outcome data that are continuously updated.

International studies of the role of technology on rapidly increasing healthcare costs have paid particular attention to the cost implications of new CARP technologies in CHD. However, while the short-term costs of CARP in CHD can be readily determined, there have been no previous studies of the long-term population impact of such advances in treatment on subsequent health service cost and utilisation. This study will help define the optimal revascularisation strategy for patients in the general population. Important preplanned subgroup analysis will also provide valuable guidance on the comparative effectiveness of PCI and CABG.

\section{Author affiliations}

${ }^{1}$ School of Population Health, University of Western Australia, Crawley, Australia

${ }^{2}$ Cardiology Department, Royal Perth Hospital, Perth, Western Australia, Australia

${ }^{3}$ Cardiology Department, Fremantle Hospital, Fremantle, Western Australia, Australia

${ }^{4}$ Cardiothoracics Department, Sir Charles Gairdner Hospital, Nedlands, Western Australia, Australia

${ }^{5}$ Beth Israel Deaconess Medical Centre, Harvard Medical School, Boston, Massachusetts, USA

Acknowledgements The authors thank: (1) the staff at the Western Australian Data Linkage Branch, and data custodians of the Department of Health Inpatient Data Collections and Registrar General for access to and provision of the linked data; (2) Steve Ridout for data management; (3) the data custodians of cardiology, cardiothoracic surgery and perfusion registries (Eric Semillion, Terry Godsell, Cheryl Blanton, Sean Sng, Vincent Rajkumar, Stuart Prince, Matthew Sheminant, Van Le); (4) PathWest, Western Diagnostic Pathology, Healthscope and St John of God Pathology for providing biochemistry and blood transfusion data; (5) Trisha Denton, Lily Titmus, Monica Campbell, Melanie Betts, Marlene Kolybaba and Samantha Croom for their work in collecting procedural data from private hospitals for the PCl cohort; and (6) cardiology fellows/trainees for collection of data on stent thrombosis. Site investigators The following are acknowledged in their role as site investigators from the various hospitals involved in this study: (1) Dr Eric Whitford and $\mathrm{Dr}$ Michael Muhlmann (cardiology), Sir Charles Gairdner Hospital, Nedlands, Western Australia; (2) Dr Randall Hendriks (cardiology), St John of God Hospital, Murdoch, Western Australia; (3) Dr Bernard Hockings (cardiology), Mount Hospital, Perth Western Australia; (4) Mr Robert Larbalestier (cardiothoracic surgery), Royal Perth Hospital, Western Australia; and (5) Mr lan Gilfillan (cardiothoracic surgery), Fremantle Hospital, Western Australia.

Collaborators Eric Whitford, Michael Muhlmann, Randall Hendriks, Bernard Hockings, Robert Larbalestier and Ian Gilfillan.

Contributors CG prepared the first draft of the manuscript, coordinated responses from the authors and is a PhD scholar on the study. FMS is the principal chief investigator and had the original idea for the study, developed the data collection databases, was the lead author for the grant application to the NHMRC, and provided major revision to the first draft of the manuscript. JMR, MNg and DC contributed to the study design and analysis methods, are supervising the stent thrombosis data collection, and will supervise various data analyses. JMR led the previous work in establishing the cohort of $\mathrm{PCl}$ and CABG cases on which the current study is based. EG provided major input into the economic analysis methods. MSTH provided major contributions to the study design and analysis methods. MWK provided major input into the study design and, in particular, the analytical methods. TGB contributed to the study design. MN contributed to the study design and assisted in the logistics of data collection for CABG procedures. All authors reviewed and provided comments for the draft manuscripts, and read and gave approval for release of the final manuscript.
Funding This study is supported by the National Health and Medical Research Council of Australia (project grant 1011995).

\section{Competing interests None.}

Ethics approval The study complies with the Declaration of Helsinki and received approval from human research ethics committees of the University of Western Australia (RA/4/1/4576), Western Australian Department of Health (2011/88) and all participating hospitals. Being a large population-based epidemiological study, approval included a waiver of informed consent.

Provenance and peer review Not commissioned; internally peer reviewed.

Data sharing statement We will consider requests for data sharing on an individual basis, with an aim to sharing data whenever possible for appropriate research purposes. However, the research project uses secondary (third party) data derived from Australian (State or Federal) government registries, which are ultimately governed by their ethics committees and data custodians. Therefore, any requests to share these data will be subject to formal approval from their ethics committees overseeing the use of these data sources, along with the data custodian(s) for the data of interest.

Open Access This is an Open Access article distributed in accordance with the Creative Commons Attribution Non Commercial (CC BY-NC 4.0) license, which permits others to distribute, remix, adapt, build upon this work noncommercially, and license their derivative works on different terms, provided the original work is properly cited and the use is non-commercial. See: http:// creativecommons.org/licenses/by-nc/4.0/

\section{REFERENCES}

1. Daemen J, Boersma E, Flather M, et al. Long-term safety and efficacy of percutaneous coronary intervention with stenting and coronary artery bypass surgery for multivessel coronary artery disease. Circulation 2008;118:1146-54.

2. Hannan EL, Racz MJ, Walford G, et al. Long-term outcomes of coronary-artery bypass grafting versus stent implantation. $N$ Engl J Med 2005;352:2174-83.

3. Stroupe KT, Morrison DA, Hlatky MA, et al. Cost-effectiveness of coronary artery bypass grafts versus percutaneous coronary intervention for revascularization of high-risk patients. Circulation 2006;114:1251-7.

4. Holmes DR Jr, Leon MB, Moses JW, et al. Analysis of 1-year clinical outcomes in the SIRIUS trial: a randomized trial of a sirolimus-eluting stent versus a standard stent in patients at high risk for coronary restenosis. Circulation 2004;109:634-40.

5. Stone GW, Ellis SG, Cox DA, et al. One-year clinical results with the slow-release, polymer-based, paclitaxel-eluting TAXUS stent: the TAXUS-IV trial. Circulation 2004;109:1942-7.

6. Camenzind E, Steg PG, Wijns W. Stent thrombosis late after implantation of first-generation drug-eluting stents: a cause for concern. Circulation 2007;115:1440-55.

7. Nordmann AJ, Briel M, Bucher HC. Mortality in randomized controlled trials comparing drug-eluting vs. bare metal stents in coronary artery disease: a meta-analysis. Eur Heart $J$ 2006;27:2784-814.

8. Kastrati A, Mehilli J, Pache J, et al. Analysis of 14 trials comparing sirolimus-eluting stents with bare-metal stents. $N$ Engl J Med 2007;356:1030-9.

9. Cutlip DE, Windecker S, Mehran R, et al. Clinical end points in coronary stent trials: a case for standardized definitions. Circulation 2007;115:2344-51.

10. Mauri L, Hsieh W-h, Massaro JM, et al. Stent thrombosis in randomized clinical trials of drug-eluting stents. $N$ Engl J Med 2007;356:1020-9.

11. Spaulding C, Daemen J, Boersma E, et al. A pooled analysis of data comparing sirolimus-eluting stents with bare-metal stents. $N$ Engl $J$ Med 2007;356:989-97.

12. Stone GW, Moses JW, Ellis SG, et al. Safety and efficacy of sirolimus- and paclitaxel-eluting coronary stents. $N$ Engl J Med 2007;356:998-1008.

13. James SK, Stenestrand U, Lindback J, et al. Long-term safety and efficacy of drug-eluting versus bare-metal stents in Sweden. $N$ Engl J Med 2009;360:1933-45.

14. Mauri L, Silbaugh TS, Wolf RE, et al. Long-term clinical outcomes after drug-eluting and bare-metal stenting in Massachusetts. Circulation 2008;118:1817-27.

15. Tu JV, Bowen J, Chiu M, et al. Effectiveness and safety of drug-eluting stents in Ontario. N Engl J Med 2007;357:1393-402. 
16. Siddiqi OK, Faxon DP. Very late stent thrombosis: current concepts. Curr Opin Cardiol 2012;27:634-41.

17. Räber L, Magro M, Stefanini GG, et al. Very late coronary stent thrombosis of a newer-generation everolimus-eluting stent compared with early-generation drug-eluting stents: a prospective cohort study. Circulation 2012;125:1110-21.

18. Joner M, Nakazawa G, Finn AV, et al. Endothelial cell recovery between comparator polymer-based drug-eluting stents. J Am Coll Cardiol 2008;52:333-42.

19. Nakazawa G, Finn AV, Joner M, et al. Delayed arterial healing and increased late stent thrombosis at culprit sites after drug-eluting stent placement for acute myocardial infarction patients: an autopsy study. Circulation 2008;118:1138-45.

20. Australian Institute of Health and Welfare. Health-care expenditure on cardiovascular diseases 2008-09. Cat. no. CVD 65 ed. Canberra: AlHW, 2014

21. Bravata DM, Gienger AL, McDonald KM, et al. Systematic review: the comparative effectiveness of percutaneous coronary interventions and coronary artery bypass graft surgery. Ann Intern Med 2007:147:703-16.

22. Serruys P, Morice M-C, Kappetein A, et al. Percutaneous coronary intervention versus coronary-artery bypass grafting for severe coronary artery disease. N Engl J Med 2009;360:961-72.

23. Daemen J, Kuck KH, Macaya C, et al. Multivessel coronary revascularization in patients with and without diabetes mellitus: 3-year follow-up of the ARTS-II (Arterial Revascularization Therapies Study-Part II) Trial. J Am Coll Cardiol 2008;52:1957-67.

24. Farkouh ME, Dangas G, Leon MB, et al. Design of the Future REvascularization Evaluation in patients with Diabetes mellitus: Optimal management of Multivessel disease (FREEDOM) Trial. Am Heart J 2008:155:215-23.

25. Taggart DP. Coronary artery bypass graft vs. percutaneous coronary angioplasty: CABG on the rebound? Curr Opin Cardiol 2007:22:517-23.

26. Holman C, Bass A, Rouse I, et al. Population-based linkage of health records in Western Australia: development of a health services research linked database. Aust N Z J Public Health 1999;23:453-9.

27. Sanfilippo F, Rankin J, Hobbs M, et al. Impact of the introduction of drug eluting stents on clinical outcomes in patients undergoing percutaneous and surgical coronary artery revascularisation procedures in Western Australia. BMC Cardiovasc Disord 2013;13:47.
28. Charlson ME, Pompei P, Ales KL, et al. A new method of classifying prognostic comorbidity in longitudinal studies: development and validation. J Chronic Dis 1987;40:373-83.

29. Romano PS, Roos LL, Jollis JG. Adapting a clinical comorbidity index for use with ICD-9-CM administrative data: differing perspectives. J Clin Epidemiol 1993;46:1075-9.

30. Australian Bureau of Statistics. Census of population and housing: socio-economic indexes for areas, Australia. Canberra: Australian Bureau of Statistics, 2001.

31. Australian Bureau of Statistics. Outcomes of ABS views on remoteness consultation, Australia. Canberra: Australian Bureau of Statistics, 2001.

32. National Health and Medical Research Council. How to compare the costs and benefits: evaluation of the economic evidence. Canberra, 2001. http://www.nhmrc.gov.au/publications/synopses/_ files/cp73.pdf

33. Australian Institute of Health and Welfare. Australian refined diagnosis-related groups (AR-DRG) data cubes, 2013. http://www. aihw.gov.au/hospitals-data/ar-drg-data-cubes/

34. Rosebaum PR, Rubin DB. The central role of the propensity score in observational studies for causal effects. Biometrika 1983;70:41-55.

35. Richardson J, Robertson IK, Hobbs M, et al. The impact of new technology on the treatment and outcomes of acute myocardial infarction in Australia: incentives for technology change and their consequences for treatment decision-making. In: McClellan MB, Kessler DP, eds. Technological change in health care: a global analysis of heart attack. Ann Arbor: The University of Michigan Press, 2002:121-55.

36. Etzioni RD, Feuer EJ, Sullivan SD, et al. On the use of survival analysis techniques to estimate medical care costs. J Health Econ 1999;18:365-80.

37. Barber J, Thompson S. Multiple regression of cost data: use of generalised linear models. J Health Serv Res Policy 2004;9:197-203.

38. Pasta DJ, Taylor JL, Henning JM. Probabilistic sensitivity analysis incorporating the bootstrap: an example comparing treatments for the eradication of Helicobacter pylori. Med Decis Making 1999:19:353-63.

39. Claxton K, Sculpher M, McCabe C, et al. Probabilistic sensitivity analysis for NICE technology assessment: not an optional extra. Health Econ 2005;14:339-47. 